\title{
Comparison of the visual performance between Oculentis MF30 and Tecnis ZMB00 multifocal intraocular lenses
}

\author{
Lingying Ye ${ }^{1}$, Tianyu Chen ${ }^{2}$, Zhixiang $\mathrm{Hu}^{2}$, Qingwen Yang ${ }^{1}$, Qiudong $\mathrm{Su}^{1}$, Jin $\mathrm{Li}^{1}$ \\ ${ }^{1}$ Cataract Department, Eye Hospital of Wenzhou Medical University, Wenzhou, China; ${ }^{2}$ Retina Department, Eye Hospital of Wenzhou Medical \\ University, Wenzhou, China \\ Contributions: (I) Conception and design: J Li, L Ye; (II) Administrative support: J Li; (III) Provision of study materials or patients: All authors; (IV) \\ Collection and assembly of data: L Ye, T Chen, Z Hu; (V) Data analysis and interpretation: All authors; (VI) Manuscript writing: All authors; (VII) \\ Final approval of manuscript: All authors. \\ Correspondence to: Jin Li. Cataract Department, Eye Hospital of Wenzhou Medical University, 270\# West Xueyuan Road, Wenzhou 325000, China. \\ Email: tracy830605@126.com.
}

Background: To compare the visual performance of MF30 asymmetric refractive multifocal intraocular lenses (MIOLs) with ZMB00 all optic zone diffractive MIOLs.

Methods: This is a prospective study. Patients that underwent phacoemulsification were divided into two groups according to the type of MIOLs used: 35 patients were implanted with asymmetric refractive MIOLs and 35 patients with all optic zone diffractive MIOLs. Visual acuity (VA), refraction, defocus curves, objective optical quality, and a questionnaire evaluating quality of life were measured at 3 months postoperatively.

Results: There were no significant differences between the two groups in uncorrected distance visual acuity (UDVA), uncorrected near visual acuity (UNVA), best-corrected distance visual acuity (BCDVA), or distance-corrected near visual acuity (DCNVA). However, the uncorrected intermediate VA was $0.24 \pm 0.10$ in the refractive group and $0.31 \pm 0.13$ in the diffractive group $(\mathrm{P}<0.05)$, and the distance-corrected intermediate VA was $0.22 \pm 0.09$ in the refractive group and $0.31 \pm 0.14$ in the diffractive group $(\mathrm{P}<0.05)$. Defocus curves showed two peaks of maximum vision in both groups. However, the curve between the two peaks in the refractive group was smoother than that of the diffractive group. The modulated transfer function cut-off frequency was $22.74 \pm 12.29 \mathrm{c} / \mathrm{d}$ in the refractive group and $30.50 \pm 10.04 \mathrm{c} / \mathrm{d}$ in the diffractive group $(\mathrm{P}<0.05)$. The Optical Quality Analysis System (OQAS) values 100\% (OV100\%) was $0.75 \pm 0.41$ in the refractive group and $1.02 \pm 0.34$ in the diffractive group $(\mathrm{P}<0.05)$, while the OV20\% was $0.52 \pm 0.34$ in the refractive group and $0.71 \pm 0.25$ in the diffractive group $(\mathrm{P}<0.05)$. There was no significant difference between the two groups in overall satisfaction, spectacle independence ratio, or visual interference phenomenon.

Conclusions: Both MIOLs achieve good VA at distance and near vision. Oculentis MF30 showed better intermediate VA, and Tecnis ZMB00 appears to have better objective visual quality.

Trial registration: NCT02234635 (http://www.clinicaltrials.gov).

Keywords: Asymmetric; refractive; diffractive; multifocal intraocular lens; visual quality

Submitted Nov 11, 2020. Accepted for publication Dec 26, 2020.

doi: $10.21037 / \mathrm{atm}-20-7777$

View this article at: http://dx.doi.org/10.21037/atm-20-7777

\section{Introduction}

Multifocal intraocular lenses (MIOLs) have become a widely accepted option in cataract surgery. All MIOLs developed thus far with reported clinical outcomes are based on the principles of diffraction and refraction. With these technologies, incoming light rays are distributed onto near and distant focal points or onto several focal points in order to provide patients with good vision at all distances 
after cataract surgeries (1-7). However, these designs lead to several consequences, such as a loss of light, which may result in reduced contrast sensitivity, permanent overlapping images generating halos and glare, and a loss of image quality (8-11).

Clinically, refractive and diffractive MIOLs have proven their own advantages and side effects $(2,4)$. However, few studies have been carried out to compare these two types of MIOLs. Some researchers believe that for refractive MIOL, after eliminating the diffraction ring, the energy loss of light will be less, which is more beneficial to the improvement of visual quality (12-14). Furthermore, according to the newest design theory of refractive MIOLs, excellent objective and subjective visual acuity (VA) can be achieved (2,3,15-17).

The aim of this study is to compare the visual quality of the Oculentis MF30 refractive rotationally asymmetrical MIOLs with the Tecnis ZMB00 full optic zone diffractive MIOLs using both objective and subjective methods, and to provide a comprehensive reference for the individualized selection of MIOLs in clinics.

We present the following article in accordance with the TREND reporting checklist (available at http://dx.doi. org/10.21037/atm-20-7777).

\section{Methods}

This non-randomized, observational study was performed at the Eye Hospital of Wenzhou Medical University (Zhejiang, China), and was approved by the institutional review board and ethics committee of Wenzhou Medical University (No. KYK-2015-20). Practices and research were conducted in accordance with the Declaration of Helsinki (as revise in 2013), and informed consent was obtained from all patients prior to participation.

Patient records between October 2016 and August 2017 were evaluated, and 70 patients (70 eyes) that had undergone phacoemulsification and IOL implantation surgery were included. Thirty-five patients were implanted with refractive rotationally asymmetrical MIOLs (Oculentis MF30) and 35 patients were implanted with all optic zone diffractive MIOL (Tecnis ZMB00). The inclusion criteria were as follows: (I) patients older than 50 year of age with age-related cataract; (II) axial length between 21 and $25 \mathrm{~mm}$; and (III) IOL was implanted in the capsular bag. Patients were excluded based on the following criteria: (I) patients less than 50 years of age; (II) patients with corneal astigmatism of more than $1.0 \mathrm{D}$; (III) patients with cornea lesions or scars, macular diseases, ocular inflammation, or any other ocular diseases; and (IV) patients with any ocular surgical history.

If a patient had undergone binocular cataract surgeries, the first operated eye was selected. Visual function data was collected by clinical researchers who did not know the type of IOL that the patient had received.

\section{Patient assessment}

All patients received a full pre- and post-operative ophthalmologic assessment. The preoperative examination included refraction, uncorrected distance visual acuity (UDVA), best-corrected distance visual acuity (BCDVA), slit-lamp examination (SL115; Carl Zeiss, Oberkochen, Germany), fundus examination under dilation, corneal topography (Pentacam, Oculus, Wetzlar, Germany), endothelial cell count (SP 2000P specular microscope, Topcon, Norway, Europe BV), biometry (IOLMaster, Carl Zeiss Meditec AG, Jena, Germany), and retinal optical coherence tomography (Cirrus 4000 OCT, Carl Zeiss Meditec AG, Jena, Germany). Patients were evaluated at 1-day, 1-week, and 1- and 3-month. At each followup visit, the UDVA and BCDVA, uncorrected near visual acuity (UNVA) and distance-corrected near visual acuity (DCNVA), uncorrected intermediate visual acuity (UIVA) and distance-corrected intermediate visual acuity (DCIVA), refraction, and Optical Quality Analysis System (OQAS) II were measured. At the 3-month evaluation, the defocusing curve was measured and patients were requested to complete a purpose-developed satisfaction questionnaire.

\section{Intraocular lens}

The Lentis Mplus LS-313 MF30 (Oculentis GmbH, Berlin, Germany) (Figure 1A) is an asymmetric refractive MIOL that has been extensively used. This refractive MIOL is designed with a $6.0 \mathrm{~mm}$ optic and a sector-shaped 3.0 diopter (D) near segment. The special principle of this MIOL is that the light is not refracted symmetrically around the optical axis. The ZMB00 (Advanced Medical Optics, Santa Ana, CA, USA) (Figure 1B) is a diffractive MIOL with $4.0 \mathrm{D}$ near segment. It has a full diffractive optic in the posterior surface with a special margin design that is round in the front portion and square in the back. Optical analog diagrams stimulate the ray tracing of these two MIOLs and show that the MF30 model has a long and narrow blur spot (Figure $2 A$ ), while the ZMB00 model has a relatively short and wide blur spot (Figure $2 B$ ), indicating 

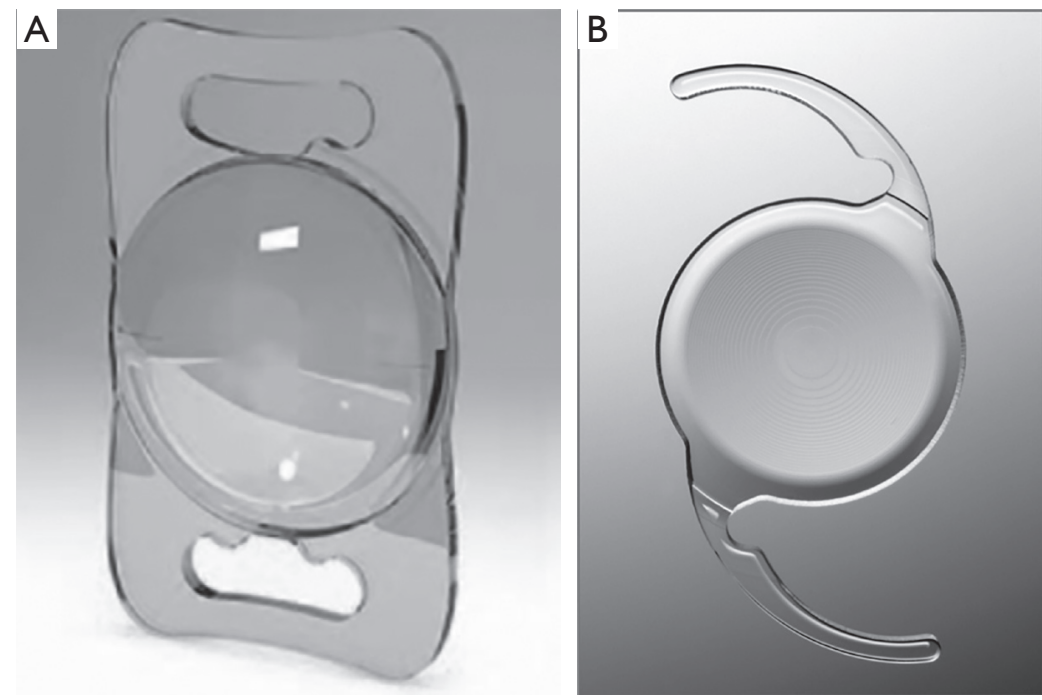

Figure 1 Two types of multifocal IOLs. (A) Lentis Mplus LS-313 MF30 multifocal IOL; (B) ZMB00 multifocal IOL. IOL, intraocular lens.
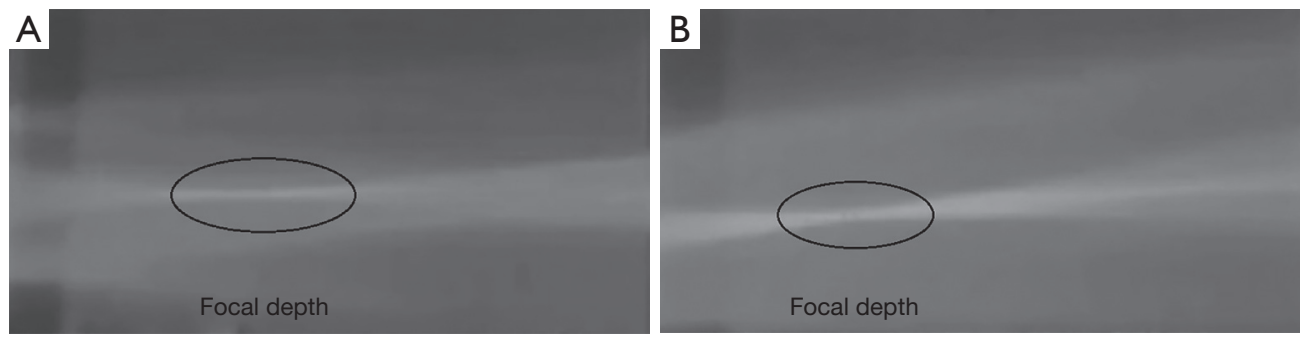

Figure 2 Ray tracing diagrams show the focal depth of each MIOL. (A) Lentis Mplus LS-313 MF30 multifocal IOL; (B) ZMB00 multifocal IOL. MIOL, multifocal intraocular lens; IOL, intraocular lens.

that the MF30 model may have an extended focal depth compared to the ZMB00 model.

\section{Surgical procedures}

All cataract surgeries were performed by an experienced surgeon. The surgical procedure was the same for both lenses. All surgeries were performed under topical anaesthesia using a $2.2 \mathrm{~mm}$ transparent corneal incision. Phacoemulsification was performed using the Infiniti vision system unit (Alcon Laboratories, Inc., Fort Worth, Texas, USA). Polishing of the posterior capsule was also performed. The IOL was implanted in the capsular bag.

\section{Outcome measurements}

The UDVA and BCDVA of each selected eye were measured at $5 \mathrm{~m}$ using a standard logarithmic VA chart with an illumination of $80 \mathrm{~cd} / \mathrm{m}^{2}$. UNVA and DCNVA were measured at a reading distance of $33 \mathrm{~cm}$ using a near logarithmic VA chart. UIVA and DCIVA were measured at a distance of $80 \mathrm{~cm}$ using a logarithmic VA chart. Both intermediate and near VA were measured under photopic conditions with an illumination of $80 \mathrm{~cd} / \mathrm{m}^{2}$. VA results were recorded in logarithm of the minimum angle of resolution (logMAR).

OQAS II is a system based on a double-pass technique to analyze the visual quality in human eyes. It analyzes all optical information of a surface, taking into account the influence of scattering, aberration, and diffraction, and obtains the correct point spread function (PSF) image. OQAS is currently the only available system to objectively analyze visual quality $(18,19)$. In this study, OQAS II was used to obtain the ocular objective optical quality 
Table 1 Clinical and demographic data of patients implanted with refractive and diffractive MIOLs (VA results were recorded in $\log \mathrm{MAR})$

\begin{tabular}{lccc}
\hline Basic information & $\begin{array}{c}\text { Refractive } \\
\text { MIOL }\end{array}$ & $\begin{array}{c}\text { Diffractive } \\
\text { MIOL }\end{array}$ & P value \\
\hline Age & $69.48 \pm 8.09$ & $68.41 \pm 8.77$ & 0.331 \\
Gender (male/female) & $20 / 15$ & $18 / 17$ & 0.189 \\
Preoperative VA & $0.51 \pm 0.22$ & $0.51 \pm 0.21$ & 0.478 \\
\hline
\end{tabular}

Data are expressed as the mean \pm standard deviation (SD) or median (range). Differences between the groups were analyzed by independent $t$ test for normally distributed data and the Mann-Whitney $U$ test for non-normally distributed data. Categorical data was compared using the Chi-squared $\left(\chi^{2}\right)$ test. MIOL, multifocal intraocular lens; logMAR, logarithm of the minimum angle of resolution; VA, visual acuity.

parameters 3 months after IOL implantation, including the modulation transfer function (MTF) cut-off frequency, objective scatter index (OSI), Strehl ratio (SR), and OQAS values (OVs $100 \%, 20 \%$ and $9 \%$ ). MTF is the ratio of contrast between the retinal image and the original object. The MTF cut off indicates the corresponding spatial frequency when the MTF value is 0.01 . A normal MTF value is larger than 30 , with larger values indicating better visual quality (20). The OV $100 \%$, OV20\%, and OV9\% refer to the OQAS value calculated by the system at three contrasts, which are commonly used in ophthalmic practice. Therefore, these three OVs are closely related to the MTF curve. SR describes the area under the MTF curve and reflects the sum of low, medium, and high frequency information. The value range is $0-1$; the closer this value is to 1 , the smaller the aberration. OSI is an index of intraocular scattered light $(19,21)$. The higher the value of the MTF cut off, SR, and OVs, and the lower the OSI, the better the objective optical quality. The instrument was set to measure with an artificial pupil diameter of $4.0 \mathrm{~mm}$, which is a standard size used in clinical double-pass studies (22).

Defocus curves were used to analyze the correlations between $\log$ MAR VA and image quality measures for the two MIOLs $(23,24)$. This was evaluated at $5 \mathrm{~m}$ using a standard logarithmic VA chart. Defocus was performed from 1.0 to $-4.0 \mathrm{D}$ in $0.5 \mathrm{D}$ steps starting from the best correction at distance (25).

The subjective visual quality of postoperative patients was investigated using a Chinese version of the National Eye Institute Visual Function Questionnaire (CHI-VFQ-25) (26), including subjective visual evaluation, spectacle independence ratio, and visual disturbance symptoms. Patients' subjective visual quality was evaluated on a scale of 0 to 10 points, with 10 points being full marks and representing the highest visual quality. To calculate the rate of spectacle independence, the need for frame glasses or corneal contact lenses for distance, medium, and near vision after surgery was recorded. Symptoms of visual interference, such as glare and halo, were also investigated in the questionnaire.

\section{Data analysis}

Statistical analysis was performed using SPSS software for Windows (version 19.0, SPSS Inc., Chicago, IL, USA). The Chi-squared $\left(\chi^{2}\right)$ test and Spearman rank correlation was used for categorical data, the $t$ test and Pearson correlation were used for measurement data, and the Mann-Whitney $\mathrm{U}$ test and Spearman correlation were used when a normal distribution was not expected. Results are presented as the mean \pm standard deviation (SD). Differences with $\mathrm{P}<0.05$ were considered to be statistically significant.

Sample size was determined using $\mathrm{G}^{*}$ Power software. Before the formal experiment, we measured the MTF values of 20 patients (10 from each group) to calculate the sample size. We assumed $\alpha=0.05$ and $\beta=0.95$ in the calculation and found the final sample size for the experiment was $\mathrm{n} 1=\mathrm{n} 2=32$ after using the $\mathrm{G}^{*}$ Power sample size calculation software to analyze the results.

\section{Results}

\section{General data}

All patients completed examinations at 3 months after cataract surgery. No intraoperative or postoperative complications occurred during the study. The preoperative conditions of patients in these two groups were analyzed including age, gender, and preoperative VA. No significant differences were found (shown in Table 1).

\section{Visual and refractive outcomes}

The postoperative visual and refractive outcomes are summarized in Table 2. No statistically significant differences were observed between the two groups in UDVA, UNVA, BCDVA, or DCNVA. However, the UIVA was $0.24 \pm 0.10$ in the refractive group and $0.31 \pm 0.13$ in the diffractive group $(\mathrm{P}<0.05)$, and the DCIVA was $0.22 \pm 0.09$ in 
Table 2 Postoperative visual and refractive outcomes in the refractive and diffractive groups at 3 months postoperatively (results were recorded in $\log M A R)$

\begin{tabular}{lccc}
\hline Measure & $\begin{array}{c}\text { Refractive } \\
\text { MIOL }\end{array}$ & $\begin{array}{c}\text { Diffractive } \\
\text { MIOL }\end{array}$ & P value \\
\hline Distance & & & \\
Uncorrected & $0.06 \pm 0.07$ & $0.09 \pm 0.09$ & 0.236 \\
Best-corrected & $0.05 \pm 0.06$ & $0.03 \pm 0.05$ & 0.337 \\
Intermediate & & & \\
Uncorrected & $0.24 \pm 0.10$ & $0.31 \pm 0.13$ & $0.029^{*}$ \\
Distance-corrected & $0.22 \pm 0.09$ & $0.31 \pm 0.14$ & $0.016^{*}$ \\
Near & & & \\
Uncorrected & $0.23 \pm 0.12$ & $0.24 \pm 0.14$ & 0.800 \\
Distance-corrected & $0.21 \pm 0.11$ & $0.20 \pm 0.12$ & 0.866 \\
\hline
\end{tabular}

Data are expressed as the mean \pm SD or median (range). Differences between the groups were analyzed by independent $t$ test for normally distributed data and the Mann-Whitney $U$ test for non-normally distributed data. *, statistically significant difference between the groups ( $P$ value $<0.05$ ). IogMAR, logarithm of the minimum angle of resolution; MIOL, multifocal intraocular lens.

Table 3 Objective measures by OQAS II in the refractive and diffractive groups at 3 months postoperatively (the pupil is $4 \mathrm{~mm}$ )

\begin{tabular}{lccc}
\hline OQAS items & $\begin{array}{c}\text { Refractive } \\
\text { MIOL }\end{array}$ & $\begin{array}{c}\text { Diffractive } \\
\text { MIOL }\end{array}$ & P value \\
\hline MTF cut-off $(\mathrm{cpd})$ & $22.74 \pm 12.29$ & $30.50 \pm 10.04$ & $0.014^{*}$ \\
OSI & $2.52 \pm 1.28$ & $2.16 \pm 0.98$ & 0.294 \\
Strehl ratio & $0.14 \pm 0.08$ & $0.17 \pm 0.06$ & 0.104 \\
OV100\% & $0.75 \pm 0.41$ & $1.02 \pm 0.34$ & $0.011^{*}$ \\
OV20\% & $0.52 \pm 0.34$ & $0.71 \pm 0.25$ & $0.030^{*}$ \\
OV9\% & $0.33 \pm 0.23$ & $0.42 \pm 0.15$ & 0.100 \\
\hline
\end{tabular}

Data are expressed as the mean \pm SD or median (range). Differences between the groups were analyzed by independent $t$ test for normally distributed data and the Mann-Whitney $U$ test for non-normally distributed data. *, statistically significant difference between the groups ( $P$ value $<0.05$ ). OQAS, optical quality analysis system; MTF, modulation transfer function; MIOL, multifocal intraocular lens; OSI, objective scatter index; SR, Strehl ratio; OV, OQAS value.

the refractive group and $0.31 \pm 0.14$ in the diffractive group $(\mathrm{P}<0.05)$ at 3 months postoperatively. Significantly better UIVA and DCIVA were found in the refractive group (Table 2).

\section{Objective optical quality outcomes}

No significant differences were observed in the OSI, SR, or OV9\% between the two groups. The MTF cutoff frequency was $22.74 \pm 12.29 \mathrm{c} / \mathrm{d}$ in the refractive group and $30.50 \pm 10.04 \mathrm{c} / \mathrm{d}$ in the diffractive group $(\mathrm{P}<0.05)$. The OV $100 \%$ was $0.75 \pm 0.41$ in the refractive group and $1.02 \pm 0.34$ in the diffractive group $(\mathrm{P}<0.05)$, and the OV20\% was $0.52 \pm 0.34$ in the refractive group and $0.71 \pm 0.25$ in the diffractive group $(\mathrm{P}<0.05)$. The MTF cut-off frequency, OV $100 \%$, and OV20\% were significantly better in the diffractive group (Table 3).

\section{Defocusing curve}

As shown in the defocusing curve, both groups provide two peaks of maximum vision. For the refractive group, one peak was at $0 \mathrm{D}$ defocus level, which corresponds to the BCDVA, and the second peak was at $-3.0 \mathrm{D}$ defocus level, which corresponds to the DCNVA. For the diffractive group, one peak was at $-0.5 \mathrm{D}$ defocus level, which corresponds to the BCDVA, and the second peak was at $-3.0 \mathrm{D}$ defocus level, which corresponds to the DCNVA.

However, the curve between the two peaks was smoother in the refractive group compared to the diffractive group, which indicates a better intermediate VA (Figure 3).

\section{Visual function (VF) questionnaire}

Ten patients $(28 \%)$ in the refractive MIOL group and eight patients $(23 \%)$ in the diffractive MIOL group developed glare and halo after surgery. There was no statistically significant difference between the two groups $(\mathrm{P}>0.05)$. Also, no significant differences were found in the spectacle independence ratios or overall satisfaction between the two groups ( $\mathrm{P}>0.05$, Table 4$)$.

\section{Discussion}

The MF30 refractive rotationally asymmetrical MIOLs and the ZMB00 full optic zone diffractive MIOLs are two popular types of MIOLs used in cataract surgery. In this study, we mainly focused on the VA performance at distant and near focal points, as well as the visual quality performance of these two MIOLs.

We found the improvement of UDVA and BCDVA in both groups to be good and similar, which is consistent with previously reported findings. These two MIOLs 


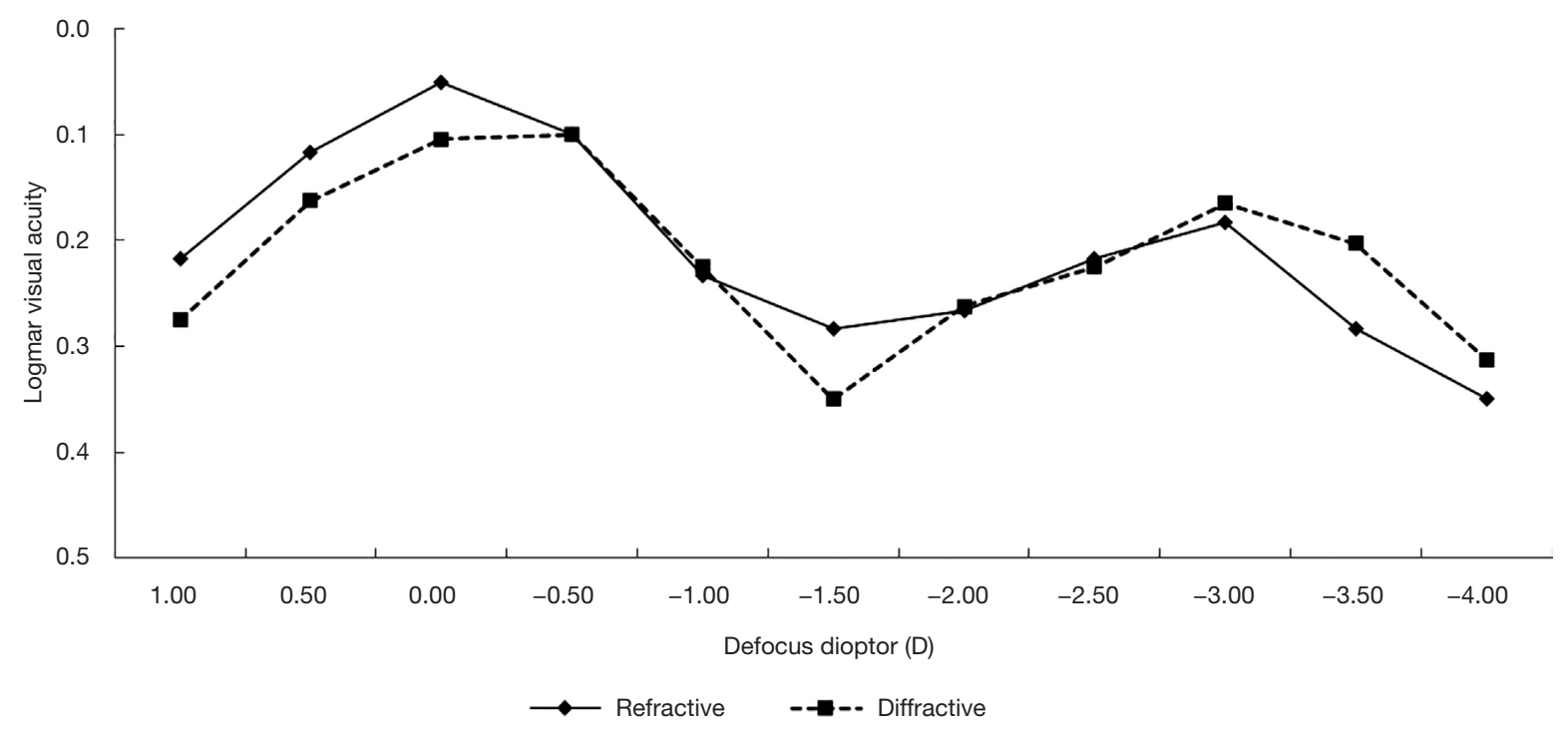

Figure 3 Defocusing curves of refractive and diffractive groups.

Table 4 Questionnaire results in the refractive and diffractive groups at 3 months postoperatively

\begin{tabular}{lccc}
\hline Subjective visual quality & $\begin{array}{c}\text { Refractive } \\
\text { MIOL }\end{array}$ & $\begin{array}{c}\text { Diffractive } \\
\text { MIOL }\end{array}$ & P value \\
\hline Glare/halo & $10 / 35$ & $8 / 35$ & 0.784 \\
$\begin{array}{l}\text { Independence spectacles } \\
\text { ratio }\end{array}$ & $21 / 35$ & $27 / 35$ & 0.240 \\
$\begin{array}{l}\text { Overall satisfaction (score } \geq 9) \\
\text { 20/35 }\end{array}$ & $21 / 35$ & 0.971 \\
\hline
\end{tabular}

Data are expressed as the mean \pm SD or median (range). Difference between groups were analyzed by $\chi^{2}$ test and Spearman rank correlation. MIOL, multifocal intraocular lens.

seem to have a similar ability to successfully restore the distance visual function after cataract surgery. Also, results showed that both groups gained good UNVA and DCNVA, indicating that both MIOLs are able to provide enough near visual function at reading distance for patients post-surgery. These results are also consistent with previous reports $(2,4,5,8,17)$. However, better results were observed in the UIVA and DCIVA of the refractive group. Defocus curves also showed a smoother curve in the refractive group than the diffractive group, which was consistent with the visual outcomes. These outcomes were expected. According to the design theory, the MF30 MIOL model is based on the concept of refractive rotational asymmetry with an inferior surface-embedded segment. The segment with the optical power is required for near vision and seamless transitions between the near and far vision zones. This design theoretically allows this MIOL to be independent of pupil size and ensures optimal adjustment of near and distance vision. Therefore, we believe that the MF30 MIOL model should provide better intermediate vision performance.

In the study, we found that the MTF cut-off frequency, OV $100 \%$, and OV20\% were significantly better in the diffractive group with $<4 \mathrm{~mm}$ pupil than the refractive group, which was not consistent with some previous studies $(19,21,22)$. In our study, all outcomes derived from the OQAS II of the diffractive group were better than the outcomes of the refractive group (as shown in Table 3). Moreover, the differences in MTF cut-off frequency, OV $100 \%$, and OV $20 \%$ between two groups were statistically significant. However, differences in OSI, SR, and OV9\% between two groups were not significant, which we believe may due to the limited case numbers. Therefore, it seems that the all optic zone diffractive MIOLs have better objective visual quality than the asymmetric refractive MIOLs. In our study, only patients with a pupil diameter of at least $4 \mathrm{~mm}$ were allowed to be measured, and we also checked its repeatability prior to initiation of the study. Therefore, we believe this subjective measurement can be used to evaluate the MIOLs and the results of our study are reliable. Moreover, the OQAS results of the diffractive MIOL in this study are very similar to the results we achieved in a previous report (5), which further highlights the reliability of the data. 
Meanwhile, no significant differences were observed in subjective visual quality between the two groups. We found no differences in the glare, halo, spectacle independence, or patient satisfaction between the groups. Previous studies have also reported that $\mathrm{ZMB} 00$ has similar subjective visual quality with the same material monofocal IOL $(1,5)$.

These findings indicate that the ZMB00 MIOL appears to provide even better objective visual quality than the MF30 MIOL. We consider that for the MF30 MIOL, although forgoing the use of diffractive rings in the optical zone may be beneficial to the use of light rays, the division of the pupil area could result in the loss of light source, which may cause impairment to objective and subjective visual quality. For ZMB00 MIOL, the material properties and the design of the MIOL may play important roles in the improvement of visual quality. Recent studies have shown that the achromatisation ability of diffractive MIOLs could provide significant improvement in polychromatic retinal image quality $(5,27)$. The diffractive MIOL model has a high Abbe number of 55.5. Therefore, the increased contrast sensitivity when compared with other materials may due to the lower chromatic dispersion, which could minimize the impact of chromatic aberration. Also, the ZMB00 MIOL uses a posterior diffractive surface to concentrate light instead of an anterior surface, which may also help to provide better focus and improve visual performance. In addition, compared with the traditional reticular margin design, ZMB00 MIOL has a special design with a round margin in the front and a square margin in the back. This may help in reducing the diffraction and refraction along the edge. We believe all these factors may contribute to the improvement of optical quality.

However, there are several limitations of this study that should be noted. This is a non-randomized study. We did not implement randomization because the patients had different visual proximity needs after cataract surgery. Therefore, we chose two MIOLs with different near additional power. Patients were assigned to the different groups according to their requirement for near vision. This is also one of the purposes of this study; we hope to offer patients a personalized choice prior to surgery, and observe whether postoperative visual performance and patient satisfaction are consistent with the preoperative choice. However, this may be a limiting factor in the study, and the different intermediate vision performance may also be attributed to the different near additional power. In future studies, we will focus on comparing these two types of MIOLs with others that have the same near additional power and randomized grouping should be performed.

\section{Conclusions}

In conclusion, we found that both MF30 and ZMB00 MIOLs are able to provide good VA at distance and near vision. The asymmetric refractive MF30 MIOLs show better intermediate VA, while the all optic zone diffractive ZMB00 MIOLs appear to have better objective visual quality. These results suggest that in addition to focusing on the improvement of the principle of focal separation, we should also pay attention to aberrations, color differences, IOL edge design, and the placement of the front and back of the focal plane. In future studies, we should analyze a larger sample of patients over a longer follow-up period.

\section{Acknowledgments}

Funding: None.

\section{Footnote}

Reporting Checklist: The authors have completed the TREND reporting checklist. Available at http://dx.doi. org/10.21037/atm-20-7777

Data Sharing Statement: Available at http://dx.doi. org/10.21037/atm-20-7777

Conflicts of Interest: All authors have completed the ICMJE uniform disclosure form (available at http://dx.doi. org/10.21037/atm-20-7777). The authors have no conflicts of interest to declare.

Ethical Statement: The authors are accountable for all aspects of the work in ensuring that questions related to the accuracy or integrity of any part of the work are appropriately investigated and resolved. Practices and research were conducted in accordance with the Declaration of Helsinki (as revise in 2013). This non-randomized, observational study was performed at the Eye Hospital of Wenzhou Medical University (Zhejiang, China), and was approved by the institutional review board and ethics committee of Wenzhou Medical University (No. KYK2015-20), and informed consent was obtained from all patients prior to participation.

Open Access Statement: This is an Open Access article 
distributed in accordance with the Creative Commons Attribution-NonCommercial-NoDerivs 4.0 International License (CC BY-NC-ND 4.0), which permits the noncommercial replication and distribution of the article with the strict proviso that no changes or edits are made and the original work is properly cited (including links to both the formal publication through the relevant DOI and the license). See: https://creativecommons.org/licenses/by-nc-nd/4.0/.

\section{References}

1. Yamauchi T, Tabuchi H, Takase K, et al. Comparison of visual performance of multifocal intraocular lenses with same material monofocal intraocular lenses. PLoS One 2013;8:e68236.

2. Alio JL, Plaza-Puche AB, Javaloy J, et al. Comparison of the visual and intraocular optical performance of a refractive multifocal IOL with rotational asymmetry and an apodized diffractive multifocal IOL. J Refract Surg 2012;28:100-5.

3. Alfonso JF, Fernandez-Vega L, Blazquez JI, et al. Visual function comparison of 2 aspheric multifocal intraocular lenses. J Cataract Refract Surg 2012;38:242-8.

4. Gatinel D, Houbrechts Y. Comparison of bifocal and trifocal diffractive and refractive intraocular lenses using an optical bench. J Cataract Refract Surg 2013;39:1093-9.

5. Chen T, Yu F, Lin H, et al. Objective and subjective visual quality after implantation of all optic zone diffractive multifocal intraocular lenses: a prospective, case-control observational study. Br J Ophthalmol 2016;100:1530-5.

6. Ruiz-Mesa R, Abengózar-Vela A, Aramburu A, et al. Comparison of visual outcomes after bilateral implantation of extended range of vision and trifocal intraocular lenses. Eur J Ophthalmol 2017;27:460-5.

7. Mcneely RN, Pazo E, Spence A, et al. Visual quality and performance comparison between 2 refractive rotationally asymmetric multifocal intraocular lenses. J Cataract Refract Surg 2017;43:1020-6.

8. Alio J, Plaza-Puche A, Férnandez-Buenaga R, et al. Multifocal intraocular lenses: An overview. Surv Ophthalmol 2017;62:611-34.

9. de Vries NE, Nuijts RM. Multifocal intraocular lenses in cataract surgery: literature review of benefits and side effects. J Cataract Refract Surg 2013;39:268-78.

10. Javitt J, Brauweiler HP, Jacobi KW, et al. Cataract extraction with multifocal intraocular lens implantation: clinical, functional, and quality-of-life outcomes. Multicenter clinical trial in Germany and Austria. J
Cataract Refract Surg 2000;26:1356-66.

11. Abdelrazek Hafez T, Helaly HA. Spectacle Independence And Patient Satisfaction With Pseudophakic MiniMonovision Using Aberration-Free Intraocular Lens. Clin Ophthalmol 2019;13:2111-7.

12. Bala C, Meades K. Improvement in vision with inverted placement of an asymmetric refractive multifocal intraocular lens. J Cataract Refract Surg 2014;40:833-5.

13. Yu S, Kim JH, Lee GJ, et al. Clinical Outcomes of Patients with Refractive Aspheric Multifocal IOL Implantation. Journal of the Korean Ophthalmological Society 2014;55:991.

14. Tchah H, Nam K, Yoo A. Predictive factors for photic phenomena after refractive, rotationally asymmetric, multifocal intraocular lens implantation. Int J Ophthalmol 2017;10:241-5.

15. Moore JE, McNeely RN, Pazo EE, et al. Rotationally asymmetric multifocal intraocular lenses: preoperative considerations and postoperative outcomes. Curr Opin Ophthalmol 2017;28:9-15.

16. McAlinden C, Moore JE. Multifocal intraocular lens with a surface-embedded near section: Short-term clinical outcomes. J Cataract Refract Surg 2011;37:441-5.

17. Venter JA, Pelouskova M, Bull CE, et al. Visual outcomes and patient satisfaction with a rotational asymmetric refractive intraocular lens for emmetropic presbyopia. J Cataract Refract Surg 2015;41:585-93.

18. Bueno JM, Perez G, Benito A, et al. Impact of scatter on double-pass image quality and contrast sensitivity measured with a single instrument. Biomed Opt Express 2015;6:4841-9.

19. Pan AP, Wang QM, Huang F, et al. Correlation among lens opacities classification system III grading, visual function index-14, pentacam nucleus staging, and objective scatter index for cataract assessment. Am J Ophthalmol 2015;159:241-7.e2.

20. Saad A, Saab M, Gatinel D. Repeatability of measurements with a double-pass system. J Cataract Refract Surg 2010;36:28-33.

21. Xu CC, Xue T, Wang QM, et al. Repeatability and reproducibility of a double-pass optical quality analysis device. PLoS One 2015;10:e0117587.

22. Vilaseca M, Arjona M, Pujol J, et al. Optical quality of foldable monofocal intraocular lenses before and after injection: comparative evaluation using a double-pass system. J Cataract Refract Surg 2009;35:1415-23.

23. Buckhurst PJ, Wolffsohn JS, Naroo SA, et al. Multifocal intraocular lens differentiation using defocus curves. Invest 
Ophthalmol Vis Sci 2012;53:3920-6.

24. Gil MA, Varón C, Cardona G, et al. Visual acuity and defocus curves with six multifocal intraocular lenses. Int Ophthalmol 2020;40:393-401.

25. Pieh S, Kellner C, Hanselmayer G, et al. Comparison of visual acuities at different distances and defocus curves. J Cataract Refract Surg 2002;28:1964-7.

26. Chan CWS, Wong D, Lam CLK, et al. Development of a Chinese version of the National Eye Institute Visual

Cite this article as: Ye L, Chen T, Hu Z, Yang Q, Su Q, Li J. Comparison of the visual performance between Oculentis MF30 and Tecnis ZMB00 multifocal intraocular lenses. Ann Transl Med 2021;9(2):144. doi: 10.21037/atm-20-7777
Function Questionnaire (CHI-VFQ-25) as a tool to study patients with eye diseases in Hong Kong. Br J Ophthalmol 2009;93:1431-6.

27. Lubinski W, Gronkowska-Serafin J, PodboraczynskaJodko K. Clinical outcomes after cataract surgery with implantation of the Tecnis ZMB00 multifocal intraocular lens. Med Sci Monit 2014;20:1220-6.

(English Language Editor: A. Kassem) 